\title{
Замещение фосфора на поверхности $\ln P(001)$ при отжиге в потоке мышьяка
}

\author{
() Д.В. Дмитриев ${ }^{1}$, Д.А. Колосовский ${ }^{1,2}$, Е.В. Федосенко ${ }^{1}$, А.И. Торопов ${ }^{1}$, К.С. Журавлев ${ }^{1,2}$ \\ ${ }^{1}$ Институт ффизики полупроводников им. А.В. Ржанова Сибирского отделения Российской академии наук, \\ 630090 Новосибирск, Россия \\ ${ }^{2}$ Новосибирский государственный университет, \\ 630090 Новосибирск, Россия \\ E-mail: ddmitriev@isp.nsc.ru
}

Поступила в Редакцию 12 апреля 2021 г.

В окончательной редакции 19 апреля 2021 г.

Принята к публикации 19 апреля 2021 г.

Методом дифракции быстрых электронов на отражение in situ изучено изменение структуры и элементного состава поверхности epi-ready $\operatorname{InP}(001)$ подложки в потоке мышьяка в сверхвысоком вакууме. Показано, что в процессе отжига окисного слоя на поверхности формируется слой $\operatorname{InP}_{1-x} \mathrm{As}_{x}$, возникающий в процессе замещения фосфора мышьяком. Установлена зависимость степени замещения от температуры и времени отжига. При температуре отжига $480^{\circ} \mathrm{C}$ степень замещения фосфора мышьяком в приповерхностном слое составляет $7 \%$, при температуре $540^{\circ} \mathrm{C}$ достигает $41 \%$. Время отжига слабо влияет на степень замещения.

Ключевые слова: фосфид индия, мышьяк, отжиг, замещение, дифракция.

DOI: 10.21883/FTP.2021.10.51437.36

\section{1. Введение}

$\operatorname{InP}(001)$ подложки активно применяются при создании гетероэпитаксиальных структур для современных приборов опто- и микроэлектроники [1-3]. Первым наиболее важным этапом эпитаксии является очистка поверхности подложки в ростовой камере и получение атомарно-чистой поверхности InP непосредственно перед эпитаксиальным ростом. Для удаления оксида с поверхности применяют термический отжиг подложки [4,5]. Значительное количество оксидов индия удаляется с поверхности при температуре $453^{\circ} \mathrm{C}$, а оксидов фосфора при $458^{\circ} \mathrm{C}$, не вызывая разложения поверхности. Однако для полного термического удаления оксидов необходимо поднимать температуру подложки выше неконгруэнтного разложения $\mathrm{InP}\left(480^{\circ} \mathrm{C}\right)[6]$. Поэтому для предотвращения разложения поверхности прогрев InP-подложек осуществляется в потоке фосфора или мышьяка. Выбор мышьяка в качестве элемента V группы обусловлен его лучшей технологичностью, так как фосфор сложно удалить из ростовой камеры, что приводит к неконтролируемому встраиванию фосфора в слои InGaAs или InAlAs и формированию четверных интерфейсных слоев. Гетерограницы таких слоев существенно менее гладкие, чем гетерограницы слоев, содержащих только мышьяк в качестве элемента V группы [7-9].

При взаимодействии мышьяка (в молекулярно-лучевой эпитаксии - МЛЭ) или мышьяксодержащих газов (в газофазовой эпитаксии из металлоорганических соединений - МОСГФЭ) с атомарно-чистой поверхностью InP при температурах $>350^{\circ} \mathrm{C}$ наблюдается полное замещение фосфора мышьяком в двух верхних бислоях и формирование слоя InAs [10-12]. При этом на поверхности реализуются реконструкционные переходы [8], аналогичные переходам, наблюдаемым при нагреве подложки InAs [13]. При взаимодействии мышьяка с окисленной поверхностью ситуация усложняется. По данным [5], поверхность подложки после удаления оксидов равномерно покрывается субмонослойным ( 5\%) слоем мышьяка, в [14] показали, что мышьяк замещает кислород в оксиде индия, а в [9] решили, что мышьяк замещает фосфор в подложке и формируется слой InAs толщиной в 1-2 бислоя, аналогично процессам на атомарно-чистой поверхности InP. При этом авторы не наблюдали за изменениями в реконструкции поверхности в процессе отжига, а фиксировали только переход в $(4 \times 2)$, считая его наличие окончанием процесса отжига. Наблюдаемые в процессе отжига подложки сверхструктуры отличаются от сверхструктур на атомарно-чистой поверхности InP, появляются новые сверхструктуры, характерные для InAs [15]. Очевидно, что присутствие слоя естественных оксидов усложняет процессы, протекающие на поверхности, между тем их понимание важно для технологии роста гетероэпитаксиальных структур, так как они влияют на начальные этапы зародышеобразования, которые непосредственно следуют за очисткой поверхности и определяют характеристики и качество синтезируемых эпитаксиальных слоев.

В данной работе изучена зависимость степени замещения фосфора мышьяком в приповерхностном слое от температуры и времени отжига epi-ready подложек $\operatorname{InP}(001)$ в потоке мышьяка. Показано, что формируемый приповерхностный слой является твердым раствором InPAs, состав которого зависит от условий отжига. 

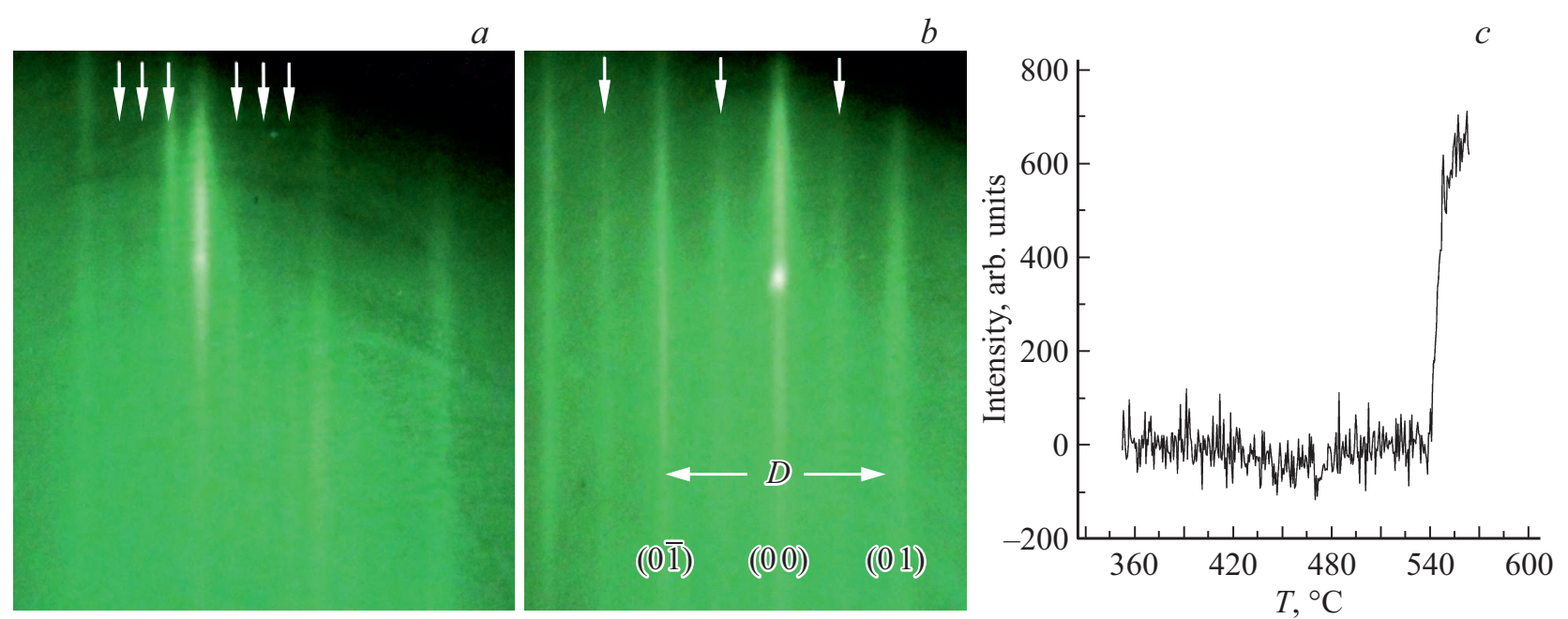

Рис. 1. Дифракционные картины сверхструктуры $(4 \times 2)$ на поверхности $\operatorname{InP}(001)$, прогретой до $540^{\circ} \mathrm{C}$ в потоке мышьяка: $a-$

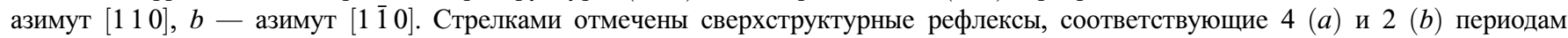
кристаллической решетки. Величина $D$ измеряется между основными рефлексами $(0 \overline{1})$ и $(01) . c-$ зависимость интенсивности сверхструктурного рефлекса $(0$ 1/4) на ДБЭО картине в азимуте [1 10$]$ от температуры отжига.

\section{2. Эксперимент}

Исследования проводились на установке МЛЭ Compact-21T фирмы Riber, оснащенной системой дифракции быстрых электронов на отражение (ДБЭО) и системой анализа дифракционных картин $\mathrm{kSA} 400$ фирмы k-Space Associates, используемой для изучения атомных процессов на поверхности. Температура подложки контролировалась in situ инфракрасным пирометром „Ircon Modline Plus“, предварительно откалиброванным по температуре реконструкционных переходов на поверхности InP [13]. Для варьирования потока мышьяка в широком диапазоне использовался вентильный источник мышьяка модели VAC500. Для измерения потока мышьяка использовался вакуумный датчик - ионизационная лампа „Bayard-Alpert JBA“, которая помещалась в положение под подложкой. Для исследований использовались epi-ready подложки $\operatorname{InP}(001)$ фирмы AXT, покрытые пассивирующим окисным слоем, упакованные в специализированную тару в атмосфере инертных газов, что позволяет избежать дополнительной подготовки подложки перед загрузкой в МЛЭ установку. Предварительная очистка подложки и держателя от легколетучих соединений, следов химической обработки и воды проводилась при температуре $150^{\circ} \mathrm{C}$ в специализированной камере установки МЛЭ, отделенной шиберным затвором от ростовой камеры. Затем окисный слой с поверхности подложки удалялся в ростовой камере МЛЭ установки прогревом до появления сверхструктуры $(4 \times 2)$ [16]. Температура отжига зависела от потока мышьяка, который варьировался в диапазоне $(0.5-5.0) \cdot 10^{-5}$ Topp. Ex situ изучение поверхности образцов проводилось методом рентгеновской фотоэлектронной спектроскопии (РФЭС). Спектры РФЭС получали с использованием аналитической сверхвысоковакуумной системы
Nanoscan-50 (Cameca). Количественные данные о составе поверхности образцов были получены с учетом коэффициента чувствительности для каждого элемента, которые были рассчитаны из теоретических сечений фотоионизации, с учетом функции пропускания анализатора спектрометра.

\section{3. Экспериментальные результаты и обсуждение}

При повышении температуры подложки $\operatorname{InP}(001)$ в потоке мышьяка происходили следующие изменения на поверхности, регистрируемые методом ДБЭО. При температуре до $T=300^{\circ} \mathrm{C}$ поверхность оставалась покрытой аморфным слоем окисла, который диффузно рассеивал электронный пучок. При температуре подложки $\sim 350^{\circ} \mathrm{C}$ окисный слой утончался, что проявлялось в уменьшении интенсивности фона дифракционной картины и проявлении $(1 \times 1)$ рефлексов кристаллической структуры подложки $\mathrm{InP}$. При $T>400^{\circ} \mathrm{C}$ происходило дальнейшее утончение окисного слоя и формирование сверхструктуры $(2 \times 3)$. При $T=450^{\circ} \mathrm{C}$ сверхструктура $(2 \times 3)$ трансформировалась в сверхструктуру $(2 \times 6)[15]$. Дальнейший прогрев подложки до $480-550^{\circ} \mathrm{C}$ приводит к формированию сверхструктуры $(4 \times 2)$. Этот переход контролировался по появлению сверхструктурных рефлексов в азимуте [1 10$]$ (рис. 1,a). На рис. 1, $c$ представлен типичный профиль изменения интенсивности сверхструктурного рефлекса (0 1/4). При достижении температуры реконструкционного перехода наблюдается резкое увеличение интенсивности рефлекса. Характерная температура $\left(T_{r}\right)$ этого реконструкционного перехода зависит от потока мышьяка. Характерные температуры остальных реконструкционных переходов от 


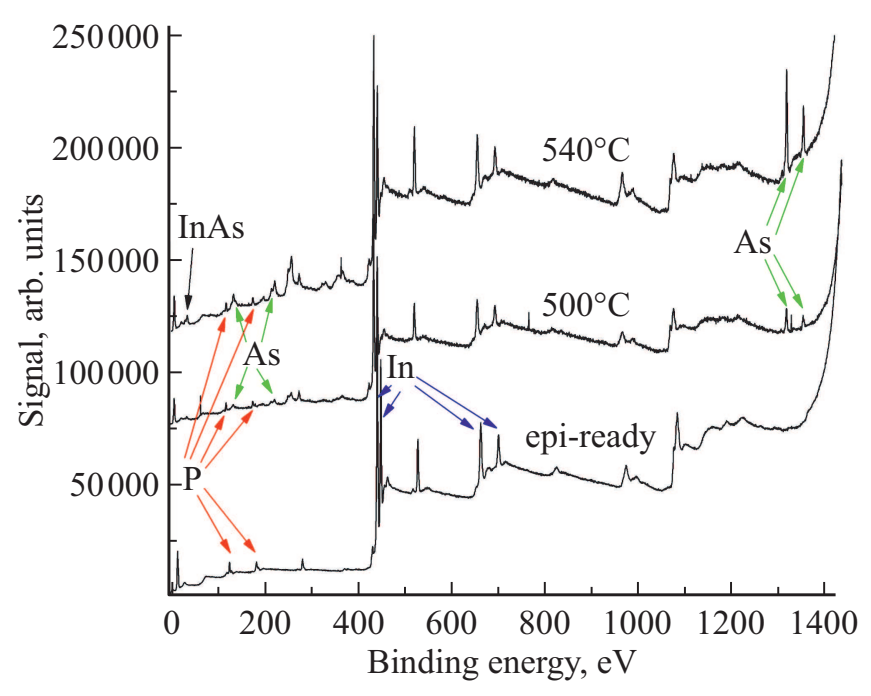

Рис. 2. Обзорный РФЭС спектр поверхности InP (снизу вверх) epi-ready, отожженной при 500 и $540^{\circ} \mathrm{C}$. Стрелками указаны характерные пики.

потока мышьяка не зависели, так как находятся ниже критической температуры разрыва связи In-P [17].

После отжига образцы остужались и переносились в РФЭС систему для анализа поверхности. Исследованы поверхности исходной epi-ready InP-подложки и после отжига при 500 и $540^{\circ} \mathrm{C}$ в потоке мышьяка. Глубина РФЭС анализа составляет 1-5 нм [18]. На обзорном спектре РФЭС (рис. 2) для всех образцов наблюдаются характерные пики, связанные с фосфором Р (130 и 184 эВ) и индием In как отдельного элемента (443.8, 665, 773 эВ), так и в составе соединения InP (444.6 эВ). На образце, отожженном при $500^{\circ} \mathrm{C}$, наблюдается появление пиков, связанных с мышьяком As (147, 204, 1323 и 1359 эВ). При увеличении температуры отжига до $540^{\circ} \mathrm{C}$ интенсивность пиков мышьяка возрастает. Отношение интенсивностей пиков As и $\mathrm{P}$ с увеличением температуры отжига увеличивается в 3.5 раза. Интенсивность пиков In изменяется незначительно. Таким образом, с увеличением температуры отжига элементный состав поверхностного слоя изменяется в сторону увеличения концентрации мышьяка. Происходит замещение десорбирующегося фосфора мышьяком и формирование слоя InPAs, состав которого зависит от температуры отжига.

Вероятно, обменная реакция P/As является причиной формирования $(2 \times 3)$ и $(2 \times 6)$ сверхструктур. Эти структуры невозможно „заморозить“ для исследований, так как они являются нестабильными и наблюдаются только при первом прогреве окисленной поверхности. В литературе наблюдали появление „3“ и „6“ в структуре поверхности на III-арсенидных материалах $[19,20]$. Отмечается, что такие структуры слабо изучены ввиду сложности их приготовления и нестабильности [21]. Вероятно, такие структуры формируются в условиях, когда на поверхности образуются вакансии V группы из-за десорбции фосфора, а темп встраивания мышьяка недостаточен из-за наличия на поверхности остаточных окислов. При дальнейшем повышении температуры формируется In-обогащенная сверхструктура $(4 \times 2)$, которая характерна только для III-арсенидных поверхностей в условиях недостатка мышьяка. При условиях формирования сверхструктуры $(4 \times 2)$ поверхность очищается от окислов и мышьяк эффективно встраивается в вакансии V подрешетки. При охлаждении подложки на поверхности формируется Аs-стабилизированная структура $(2 \times 4)$. Повторный нагрев подложки не позволяет воспроизвести условия первичного нагрева, поэтому переходы $(2 \times 3) \rightarrow(2 \times 6)$ не воспроизводятся, а реализуются реконструкционные переходы $(2 \times 4) \rightarrow(4 \times 2)$, характерные для поверхности InAs.

Появление сверхструктуры $(4 \times 2)$ наблюдается на поверхности III-арсенидных материалов (InAs, GaAs) [13]. На поверхности InP подобной структуры не наблюдается [22]. Таким образом, формирование $(4 \times 2)$ сверхструктуры связано с замещением фосфора мышьяком на поверхности InP и формированием InPAs твердого раствора.

Для определения степени замещения фосфора мышьяком в приповерхностном слое подложки InP были измерены латеральные постоянные решетки $a_{0}$ этого слоя для каждой температуры отжига. Для оценки постоянной решетки из дифракционной картины (рис. $1, b)$ измерялось расстояние $D$ между основными рефлексами $(0 \overline{1})$ и (01). Из изменения величины $D$ при отжиге определялось изменение постоянной решетки $\left(D \sim 1 / a_{0}\right)$ [23]. Для этого мы измеряли в одинаковых геометрических условиях величину $D_{(\mathrm{InP})}$ на очищаемой от окисла $\operatorname{InP}(001)$ подложке при появлении $(1 \times 1)$ сверхструктуры, которая соответствует объемной структуре, и величину $D_{(\operatorname{InAs})}$ для $\operatorname{InAs}(001)$ подложки. Значения $a_{0}$ для $\operatorname{InP}(001) \quad 0.58687$ нм и $\operatorname{InAs}(001) \quad 0.60583$ нм взяты из литературных источников [24]. Отношение разницы постоянных решетки $\Delta a_{0}=a_{0(\mathrm{InAs})}-a_{0(\mathrm{InP})}$ и расстояния между основными рефлексами $\Delta D=D_{(\mathrm{InP})}-D_{(\mathrm{InAs})}$ является коэффициентом пропорциональности $K$ для определения постоянной решетки из дифракционной картины сформированного при отжиге слоя InPAs:

$$
a_{0(\mathrm{InPAs})}=a_{0(\mathrm{InP})}+D_{(\mathrm{InPAs})} \times K[\mathrm{HM}],
$$

где $K=\Delta a_{0} / \Delta D, D_{(\mathrm{InPAs})}$ - экспериментально измеренное расстояние между тяжами $(0 \overline{1})$ и (01) для каждой температуры отжига.

Содержание мышьяка $x$ в поверхностном $\operatorname{InP}_{1-x} \mathrm{As}_{x}$ слое InP-подложки, отожженном в потоке мышьяка, оценивалось из рассчитанной $a_{0 \text { (InPAs) }}$ по закону Вегарда [25]. Для этого решалась обратная задача определения $x$ по известной $a_{0}$ :

$$
x=1-\left(\left(a_{0(\mathrm{InPAs})}-a_{0(\mathrm{InAs})}\right) /\left(a_{0(\mathrm{InP})}-a_{0(\mathrm{InAs})}\right)\right) .
$$

Экспериментально полученные значения постоянных решетки по формуле (1) и составы слоев $\operatorname{InP}_{1-x} \mathrm{As}_{x}$, 
Состав и параметр решетки $a_{0}$ приповерхностного слоя $\mathrm{InP}_{x} \mathrm{As}_{1-x}$ для разных температур отжига, определенный по данным ДБЭО

\begin{tabular}{c|c|c}
\hline $\begin{array}{c}\text { Температура } \\
\text { подложки, }{ }^{\circ} \mathrm{C}\end{array}$ & $a_{0}$, нм & Состав слоя \\
\hline 480 & 0.5880 & $\mathrm{InP}_{0.93} \mathrm{As}_{0.07}$ \\
500 & 0.5894 & $\mathrm{InP}_{0.87} \mathrm{As}_{0.13}$ \\
520 & 0.5930 & $\mathrm{InP}_{0.66} \mathrm{As}_{0.34}$ \\
540 & 0.5946 & $\mathrm{InP}_{0.59} \mathrm{As}_{0.41}$
\end{tabular}

определенные по формуле (2), приведены в таблице. Полученное изменение в составе InPAs с $13 \%$ при $500^{\circ} \mathrm{C}$ до $41 \%$ при $540^{\circ} \mathrm{C}$, т.е. в 3.2 раза, хорошо согласуется с данными, полученными методом РФЭС.

Использование литературных данных для величины $a_{0}$ бинарных соединений InP и InAs связано с тем, что точность абсолютных измерений постоянной решетки методом ДБЭО невелика $\leq 0.4 \%$ из-за необходимости использовать узкий электронный луч (диаметром $<0.1$ мм на поверхности), исследовать образец малых размеров (< 1 мм), установить расстояние между образцом и дифракционным экраном в десятки сантиметров. Точность относительных измерений высока, так как конструкция используемой МЛЭ установки Compact-21T позволяет однообразно устанавливать экспериментальные образцы и точно фиксировать угол падения электронного луча на поверхность образца, что обеспечивает воспроизводимость экспериментальных условий. Надо отметить, что эти значения получены при усреднении по глубине и площади поверхности под электронным пучком. Глубина проникновения электронного пучка в глубь

$$
T,{ }^{\circ} \mathrm{C}
$$

$180 \quad 240 \quad 300 \quad 360 \quad 420 \quad 480 \quad 540$

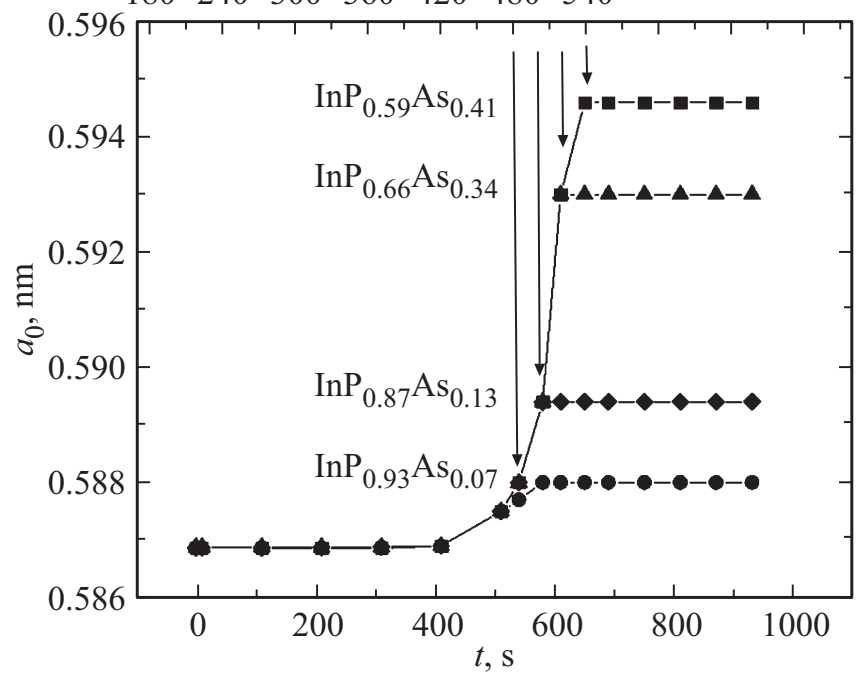

Рис. 3. Зависимость параметра решетки поверхностного слоя InPAs от температуры и времени отжига. Стрелками указаны температуры отжига образцов. кристалла при энергии электронов 12 кВ и угле падения $\sim 3^{\circ}$ составляет $\sim 1$ нм. Площадь анализируемого слоя $\sim 10 \mathrm{MM}^{2}$.

На рис. 3 представлена зависимость экспериментально измеренных латеральных параметров решетки поверхностного InPAs-слоя от температуры отжига и времени экспозиции поверхности в потоке мышьяка. Подложки нагревались со скоростью $30^{\circ} /$ мин. При температурах $>400^{\circ} \mathrm{C}$ наблюдалось утончение окисного слоя, как описано выше, и увеличение параметра решетки, связанное с замещением фосфора мышьяком и формированием InPAs-слоя. При фиксации температуры (обозначено стрелками) $a_{0}$ не увеличивалось и оставалось неизменным в широком временно́м диапазоне для всех температур отжига. Таким образом, задавая температуру отжига, можно варьировать состав и параметр решетки $a_{0}$ переходного слоя между подложкой и гетероэпитаксиальной структурой. Такой технологический прием может применяться для роста рассогласованных с подложкой InP слоев с повышенным содержанием In.

\section{4. Заключение}

В работе экспериментально изучена зависимость степени замещения фосфора мышьяком в приповерхностном слое от температуры и времени отжига epi-ready подложек $\operatorname{InP}(001)$ в потоке мышьяка. Методами РФЭС и ДБЭО показано, что формируемый приповерхностный слой является твердым раствором InPAs, состав которого зависит от температуры отжига и не зависит от времени экспозиции поверхности в потоке мышьяка. In situ измерена степень замешения фосфора мышьяком в зависимости от температуры отжига, которая составляет $7 \%$ при температуре отжига $480^{\circ} \mathrm{C}$ и возрастает до $41 \%$ с повышением температуры отжига до $540^{\circ} \mathrm{C}$.

\section{Финансирование работы}

Исследование выполнено при финансовой поддержке РФФИ и правительства Новосибирской области в рамках научного проекта № 20-42-540009.

\section{Конфликт интересов}

Авторы заявляют, что у них нет конфликта интересов.

\section{Список литературы}

[1] S. Lee, M. Winslow, C.H. Grein, S.H. Kodati, A.H. Jones, D.R. Fink, P. Das, M.M. Hayat, T.J. Ronningen, J.C. Campbell, S. Krishna. Sci. Rep., 10, 16735 (2020).

[2] J.A. del Alamo. Nature, 479, 317 (2011).

[3] T. Takahashi, Y. Kawano, K. Makiyama, S. Shiba, M. Sato, Y. Nakasha, N. Hara. IEEE Trans. Electron. Dev., 64, 89 (2017)

[4] J. Massies, J.P. Contour. J. Appl. Phys., 58, 386 (1985). 
[5] G.J. Davies, R. Heckingbottom, H. Ohno, C.E.C. Wood, A.R. Calawa. Appl. Phys. Lett., 37, 290 (1980).

[6] W.M. Lau, R.N.S. Sodhi, S. Ingrey. Appl. Phys. Lett., 52, 386 (1988).

[7] R.F.C. Farrow. J. Phys. D: Appl. Phys., 8, 87 (1975).

[8] C.H. Li, L. Li, D.C. Law, S.B. Visbeck, R.F. Hicks. Phys. Rev. B, 65, 205322 (2002).

[9] В.А. Кульбачинский, Л.Н. Овешников, Р.А. Лунин, Н.А. Юзеева, Г.Б. Галиев, Е.А. Климов, П.П. Мальцев. ФТП, 49 (2), 204 (2015).

[10] O.E. Tereshchenko, D. Paget, P. Chiaradia, E. Placidi, J.E. Bonnet, F. Wiame, A. Taleb-Ibrahimi. Surf. Sci., 600, 3160 (2006).

[11] G. Hollinger, D. Gallet, M. Gendry, C. Santinelli, P. Viktorovitch. J. Vac. Sci. Technol. B, 8, 832 (1990).

[12] J.M. Moison, M. Bensoussan, F. Houzay. Phys. Rev. B, 34, 2018 (1986).

[13] Q.-K. Xue, T. Hashizume, T. Sakurai. Progr. Surf. Sci., 56, 1 (1997).

[14] R. Averbeck, H. Riechert, H. Schlötterer, G. Weimann. Appl. Phys. Lett., 59, 1732 (1991).

[15] D.V. Dmitriev, N.A. Valisheva, A.M. Gilinsky, I.B. Chistokhin, A.I. Toropov, K.S. Zhuravlev. IOP Conf. Ser.: Mater. Sci. Eng., 475, 012022 (2019).

[16] S. Katsura, Y. Sugiyama, O. Oda, M. Tacano. Appl. Phys. Lett., 62, 1910 (1993).

[17] M. McElhinney, C.R. Stanley. J. Cryst. Growth, 150, 518 (1995).

[18] J. Moulder, W. Stickle, W. Sobol, K.D. Bomben. Handbook of X-Ray Photoelectron Spectroscopy (Published by PerkinElmer Corp., Phys. Electronics Division) Eden Prairie, Minnesota, USA, 1992).

[19] J. Behrend, M. Wassermeier, K.H. Ploog. Surf. Sci., 372, 307 (1997).

[20] K. Regiński, J. Muszalski, V.V. Preobrazhenskii, D.I. Lubyshev. Thin Sol. Films, 267, 54 (1995).

[21] T. Ohno. Surf. Sci., 357-358, 265 (1996).

[22] V.P. LaBella, Z. Ding, D.W. Bullock, C. Emery, P.M. Thibado. J. Vac. Sci. Techn. A, 18, 1492 (2000).

[23] L.-M. Peng, J.M. Cowley. Surf. Sci., 201, 559 (1988).

[24] I. Vurgaftman, J.R. Meyer, L.R. Ram-Mohan. J. Appl. Phys., 89, 5815 (2001).

[25] A.R. Denton, N.W. Ashcroft. Phys. Rev. A, 43, 3161 (1991).

Редактор Г.А. Оганесян

\section{Phosphorus substitution on the $\operatorname{InP}(001)$ surface during annealing in an arsenic flux}

D.V. Dmitriev ${ }^{1}$, D.A. Kolosovsky ${ }^{1,2}$, E.V. Fedosenko ${ }^{1}$, A.I. Toropov ${ }^{1}$, K.S. Zhuravlev ${ }^{1,2}$

${ }^{1}$ Rzhanov Institute of Semiconductor Physics, Siberian Branch Russian Academy of Sciences, 630090 Novosibirsk, Russia

2 Novosibirsk State University,

630090 Novosibirsk, Russia

Abstract We report experimental study of the transformation of the epi-ready $\operatorname{InP}(001)$ surface during annealing in an arsenic flux. Using the RHEED method, it was shown that an $\mathrm{InP}_{1-x} \mathrm{As}_{x}$ layer is formed on the surface. The amount of arsenic substitution for phosphorus at different annealing temperatures was determined, which is $7 \%$ at an annealing temperature of $480^{\circ} \mathrm{C}$ and increases to $41 \%$ with an increase in the annealing temperature to $540^{\circ} \mathrm{C}$. The annealing time has little effect on the degree of substitution. 